\title{
NUESTRO NORTE ES EL SUR: EL DOCTORADO LATINOAMERICANO EN EDUCACIÓN DE LA UFMG
}

\author{
JULIANA DE FÁTIMA SOUZA' \\ ORCID: https://orcid.org/0000-0002-8858-7197 \\ LÍVIA MARIA FRAGA VIEIRA" \\ ORCID: https://orcid.org/0000-0002-9036-0151 \\ LUÍSA FERNANDA MEJÍA TORO" \\ ORCID: https://orcid.org/0000-0002-8029-6694
}

I Universidade do Estado de Minas Gerais (UEMG), Belo Horizonte, MG, Brasil.

II Universidade Federal de Minas Gerais, Faculdade de Educação (UFMG), Belo Horizonte, MG, Brasil.

RESUMEN: La cooperación internacional es un componente estratégico en la consolidación de programas de postgrado de excelencia. En la dinamización del Sistema Nacional de Postgrado en Brasil se han valorizado instituciones del Norte Global, que concentra la mayoría de las universidades de clase mundial. Este texto presenta una experiencia que se diferencia de este modelo dando énfasis en la cooperación Sur-Sur y en los sentidos de la cooperación regional para la superación de problemas específicos de América Latina y el Caribe. El objeto del trabajo es el Doctorado Latinoamericano en Educación (DLA) de la UFMG, desarrollado en convenio con la Unesco y universidades de Chile, Colombia, Ecuador, Honduras, México, Perú y Venezuela. El análisis incluye documentos del programa y formularios respondidos por egresados. Puede verse que el DLA constituye un proyecto alternativo de internacionalización, más autónomo y auto concentrado, fundamentado en una concepción de justicia social y de solidaridad entre los estados.

Palabras-clave:DoctoradoLatinoamericanoenEducación. Internacionalización. Postgrado. América Latina. Profesión Docente.

JULIANA DE F. SOUZA - Doctora en Educación. Asesora de Relaciones Internacionales de la Universidade do Estado de Minas Gerais (UEMG). E-mail:<jusouzarp@gmail.com>.

LÍVIA M. F. VIEIRA - Doctora en Ciencias de la Educación. Profesora de la Facultad de Educación de la Universidade Federal de Minas Gerais. E-mail: < liviafraga59@gmail.com > .

LUÍSA F. M.TORO - Doctora en Educación. Investigadora de los grupos Inovar/Capes y BER/ColCiências Colombia. E-mail:<profesoraluisamejia@gmail.com>. 


\section{NOSSO NORTE É O SUL: UM ESTUDO DE CASO SOBRE O DOUTORADO LATINO-AMERICANO EM EDUCAÇÃO DA UFMG}

RESUMO: A cooperação internacional é um componente estratégico para a constituição e consolidação de programas de pós-graduação stricto sensu de excelência. Na dinamização do Sistema Nacional de Pós-Graduação no Brasil, valorizam-se as instituições do Norte global, onde se concentra a maior parte das universidades de classe mundial. Este artigo apresenta uma experiência que se distancia desse modelo, com foco na cooperação Sul-Sul e nos sentidos da cooperação regional para o enfrentamento de problemas específicos da América Latina e Caribe. O objeto do trabalho é o Doutorado Latino-Americano em Educação (DLA) da UFMG, que foi desenvolvido em parceria com a Unesco e universidades do Chile, Colômbia, Equador, Honduras, México, Peru e Venezuela. A análise inclui documentos do programa e questionários respondidos por egressos. Constatou-se que o DLA constitui um projeto alternativo de internacionalização, mais autônomo e autocentrado, fundamentado em uma concepção de justiça social e de solidariedade entre os Estados da região.

Palavras-chave:DoutoradoLatino-americanoemEducação.Internacionalização. Pós-graduação. América Latina. Profissão Docente.

\section{OUR NORTH IS THE SOUTH: A CASE STUDY ON THE LATIN AMERICAN DOCTORATE IN EDUCATION AT UFMG}

ABSTRACT: International cooperation is a strategic component for the constitution and consolidation of stricto sensu excellence postgraduate programs. In the strengthening of the Brazilian Postgraduate System, academic relations with the global North have been valued, where most of the world-class universities are concentrated. This article presents an experience that distances itself from this model, focusing on South-South cooperation and the meanings of regional cooperation to overcome specific problems of Latin America and the Caribbean. The subject of this work is the Latin American Doctorate in Education (DLA) at UFMG/Brazil, which was developed in partnership with Unesco and universities in Chile, Colombia, Ecuador, Honduras, Mexico, Peru and Venezuela. The analysis includes documents of the program and questionnaires answered by graduates. It was concluded that the DLA is an alternative internationalization project, with more autonomy and self-centered, oriented by a social justice conception and solidarity among countries of the region.

Key-words: Latin American Doctorate in Education. Internationalization. Postgraduate studies. Latin America. Teaching profession. 


\section{INTRODUCCIÓN}

La cooperación internacional es un componente estratégico para la constitución y consolidación de programas de postgrado de excelencia stricto sensu. Según la Coordenação de Aperfeiçoamento de Pessoal de Nivel Superior (Capes) - Agencia responsable por la evaluación de los cursos de maestría y doctorado en Brasil - la internacionalización constituye un factor de diferenciación en el nivel de desempeño de los programas de cada área, siendo atribuidas las notas máximas del proceso de evaluación exclusivamente a programas de referencia y reconocida inserción internacional.

Por un lado, la cooperación internacional es señalada como un medio que posibilita dinamizar el Sistema Nacional de pos graduación, la captación de conocimiento nuevo y la creación de un ambiente propicio para la innovación, siendo valorizada la intensificación de las relaciones con instituciones del Norte Global, donde está concentrada la mayor parte de las universidades de clase mundial. Por otro lado el fenómeno de la internacionalización es abordado desde una perspectiva de solidaridad, esperando relaciones de cooperación con otros Estados del Sur en los cuales la ciencia y la tecnología se encuentran en niveles menos avanzados que en Brasil (CAPES, 2010).

Tradicionalmente, sin embargo, los países de América Latina y Caribe (ALyC) interactúan más con universidades de los países desarrollados que con instituciones de educación superior (IES) de la propia región, como muestran los datos sobre movilidad académica y el carácter de las asociaciones para publicación internacional. En 2014, los principales destinos para alumnos de maestría y doctorado oriundos del Brasil, por ejemplo, eran universidades de Norte América o Europa Central (OCDE, 2016). En lo relacionado con los convenios que hacen los investigadores latinoamericanos y caribeños para la publicación de artículos en coautoría, también se observa la predominancia de instituciones europeas y estadounidenses (UNESCO, 2015).

Estos resultados no solo confirman que los sistemas de educación superior de estos países son más atractivos, sino que también son resultado de la política nacional de postgrado emprendida en Brasil desde 1970, que promueve prioritariamente la conexión con IES del eje Norte o, en algunos casos, las relaciones con países del Sur con los cuales es posible desarrollar la cooperación de forma simétrica, o al menos aproximada. En noviembre de 2017, al lanzar el Programa Institucional de Internacionalización (PrInt), Convocatoria n ${ }^{\circ} 41 / 2017$, que representa la mayor parte de 
la inversión de la Capes en internacionalización para los próximos cuatro años, fueran enumerados veintiséis países socios en los cuales deberán ser concentrados por lo menos el $70 \%$ de los recursos. Los únicos Estados de ALyC que se encuentran en la lista son Argentina y México, que al igual que el Brasil presentan los sistemas de postgrado más consolidados de la región.

Este tipo de instrucción puede contribuir a mantener la hierarquía entre los sistemas de educación superior alrededor del mundo y al empobrecimiento de la internacionalización Sur-Sur, que tiende a continuar ocupando un espacio secundario en los programas de postgrado stricto sensu. La colaboración entre países en desarrollo constituye una estrategia importante para enfrentar y superar problemas comunes, en un horizonte de cooperación y solidaridad entre pares. Además tiene un papel importante para promover la democratización de la educación superior en la región, la construcción de nuevos saberes y la expansión de la innovación endógena.

No se trata de oponerse a la cooperación con el Norte, a la cooperación regional entre los países e instituciones del Sur, pues eso sería un error. Son procesos con finalidad y recompensas distintas y que pueden ser fomentados paralelamente. Las universidades de los países desarrollados se encuentran en una posición de prestigio que no debe ser desconsiderada. A través de ellas América Latina y el Caribe tiene acceso al estado de arte en diferentes campos del conocimiento y puede mejorar su propio sistema académico. Sin embargo es necesario intensificar la asociación entre pares no solo como un deber ético, sino como fuente de un conocimiento propio, del cual la región necesita apropiarse para desarrollarse mejor, posicionarse en el campo científico internacional, bien como para alcanzar un mayor bien-estar social.

En este contexto, el presente artículo discute sobre una experiencia innovadora de internacionalización Sur-Sur emprendida en el ámbito de un programa de postgrado de excelencia: Doctorado Latinoamericano en Educación con énfasis en Políticas Públicas y Profesión Docente (DLA), vinculado al Programa de Pós-Graduação em Educação: Conhecimento e Inclusão Social da Universidade Federal de Minas Gerais (PPGE/UFMG) ofrecido desde 2010.

El Doctorado Latinoamericano en Educación fue construido a partir de un acuerdo firmado entre la Unesco y Universidades de Brasil, Chile, Colombia, Ecuador, Honduras, México, Perú y Venezuela. Este acuerdo tenía como visión la construcción de una estructura curricular común de postgrado stricto sensu, que 
pudiera ser ofrecida en los diferentes países vinculados, con el objetivo central de promover la formación calificada de doctores en el campo de la política educativa, con capacidad para investigar y producir conocimiento sobre la realidad regional. La integración académica consistía, en este sentido, en una estrategia para congregar investigadores de diferentes nacionalidades en torno de realidades similares, de modo que se pueda aportar para el fortalecimiento de la calidad de la educación de ALyC.

El DLA/UFMG fue analizado desde la perspectiva de estudio de caso cualitativo, teniendo como fuentes un conjunto de documentos, incluyendo actas de reuniones, relatorías y el proyecto pedagógico del referido curso. En 2016 y 2017 también fueron aplicados formularios con preguntas abiertas y cerradas a los egresados del curso, con el fin de conocer la perspectiva de sus directos beneficiarios. ${ }^{1}$

A continuación se busca rescatar la historia de creación del Doctorado Latinoamericano en Educación y las finalidades subyacentes a la propuesta; su proceso de implementación en la Universidad Federal de Minas Gerais; y discutir los méritos y desafíos del programa desde la perspectiva de los egresados.

\section{UNA CONSTRUCCIÓN COLECTIVA}

La posibilidad de organizar un doctorado en educación sobre una perspectiva regional fue discutida por primera vez en el ámbito de una reunión de la Red Kipus - Red de profesores para América Latina y el Caribe. Esta es una Red vinculada a la Oficina Regional de Educación para América Latina y el Caribe (Orealc/Unesco), que congrega instituciones formadoras de profesores de Argentina, Bolivia, Brasil, Chile, Colombia, Costa Rica, Ecuador, Honduras, México, Perú y Venezuela, con el objetivo de contribuir a la discusión sobre políticas públicas nacionales sobre docencia; así como el de apoyar la construcción de alianzas intersectoriales en el fortalecimiento de la profesión docente incentivando el protagonismo de las instituciones formadoras en las transformaciones educativas necesarias en la región. Esta red surgió como producto de los acuerdos hechos durante el Seminario Latinoamericano de Universidades Pedagógicas y sistematizados en el documento conocido como Carta de Santiago, de 2003. En esa ocasión los Rectores de Universidades Pedagógicas, en compañía de algunos expertos y líderes en educación establecen una serie de compromisos que apuntan al cumplimiento de las recomendaciones educativas promulgadas internacionalmente. ${ }^{2}$ La constitución de la Red respondería a una 
articulación real que promulgara el cumplimiento de 17 compromisos firmados en Santiago de Chile, que pretendían contribuir a la superación de desafíos de la región tales como la Paz, el fortalecimiento de una identidad latinoamericana, la erradicación del analfabetismo, mejorar el financiamiento de la educación por medio de la sensibilización de los gobiernos, promover la movilidad académica e investigación, fortalecer la docencia por medio de programas de formación y calidad de trabajo entre otros. Específicamente, la constitución de la red, responde a la puesta en marcha del punto uno y siete de dicho compromiso:

1. Desarrollar una estrategia de cooperación horizontal, fortaleciendo una alianza estratégica latinoamericana que incluya universidades pedagógicas, institutos pedagógicos, escuelas normales y facultades de educación.

7. Sentar las bases para el establecimiento de una red regional que facilite, a través de diversos mecanismos, la cooperación académica para la formación de docentes. (CARTA DE SANTIAGO, 2003, p. 138)

Adicionalmente, en el Encuentro Regional de la Red Kipus, realizado en Venezuela en octubre de 2006, fue firmada una carta de compromiso en la cual instituciones de diferentes países establecían la intención de desenvolver un programa de postgrado stricto sensu enfocado en la cuestión de la profesión docente que diera cuenta de las peculiaridades del subcontinente latino americano y caribeño. La discusión fue retomada en agosto de 2007, durante un congreso internacional sobre la evaluación de la función docente realizado en Chile, cuando miembros de la red allí presentes conformaran una nueva agenda para tratar específicamente del doctorado. La primera reunión de trabajo oficial del DLA se dio en los días 08 y 09 de octubre de 2007, en Venezuela, con el fin de discutir efectivamente la viabilidad de creación del programa, sus propósitos y su sentido. El encuentro fue organizado por la Unesco, a través de la Orealc y también del Instituto Internacional para la Educación Superior en América Latina y el Caribe (Iesalc) que había sido vinculado a la iniciativa, con la participación de representantes de las instituciones de la educación superior que se ofrecieron como voluntarios para ser sede en sus países del futuro doctorado - la Universidad Pedagógica Experimental Libertador, de Venezuela; la Universidad Pedagógica Nacional, de México; la Universidad Pedagógica Nacional, de Colombia; la Universidad Tecnológica San Antonio de Márchala, de Ecuador; la Universidad Pedagógica Nacional Francisco Morazán, de Honduras; la Pontificia Universidad Católica del Perú; la Universidade Federal de Minas Gerais, del Brasil; y cuatro instituciones chilenas 
(Universidad del Bío-Bío, Universidad de la Frontera, Universidad Metropolitana de Ciencias de la Educación e Universidad Católica Cardenal Silva Henríquez). En esa reunión se nombró un grupo técnico ${ }^{3}$ para avanzar en el diseño de la propuesta del DLA, incluyendo el compromiso de los participantes de presentar ante sus instituciones un modelo de Acuerdo de Cooperación Institucional que debería ser firmado por todos.

A partir de ahí se continuo con una intensa agenda de trabajo, con reuniones consecutivas para la elaboración y discusión de la estructura del doctorado. La segunda reunión oficial ocurrió en el Ecuador, en los días 03 y 04 de marzo de 2008; la tercera en Brasil, los días 15 y 16 de mayo de 2008; la cuarta fue realizada en el Perú, durante los días 4, 5 y 6 de junio de 2008, con la presencia también de los rectores y/o directores de las IES firmadas, bien como los representantes de la Red Kipus, de la Orelac y del Iesalc, que celebran entre sí en aquel momento el Acuerdo de Cooperación Interinstitucional; ya la quinta reunión se dio en Chile, en los días 24 y 25 de septiembre de 2008; y la sexta en los días 17 y 18 de febrero de 2009, cuando fueran aprobados la estructura curricular y las directrices generales de funcionamiento del doctorado y finalmente establecido su lanzamiento para el segundo semestre del mismo año, en acto en la Universidad Pedagógica de México entre los días 17 y 19 de septiembre.

Como consta en el programa aprobado la implementación de un programa de postgrado interinstitucional e internacional constituía una estrategia de actuación en red para el enfrentamiento de problemas comunes en la formación de formadores. El DLA se justificaba por su potencial de contribuir con: i) la consolidación del intercambio entre investigadores de países que buscan el conocimiento y la transformación de la realidad educativa latinoamericana; ii) la atención de las necesidades de formar doctores de los diversos sectores involucrados en el desarrollo de la profesión docente, iii) la generación de condiciones para el fortalecimiento de la investigación sobre $\mathrm{ALyC}$ y por consecuencia un aumento de la producción académica en el campo de la educación a partir de una perspectiva regional; y iv) la promoción y el reconocimiento de la profesión docente como un aspecto fundamental para la mejoría de la calidad de la educación (DLA, 2009, p. 9).

El derrotero formativo fue estructurado de tal manera que las universidades sedes del programa pudieran adaptarlo a sus contextos nacionales. De esa manera el plan de estudios del DLA contemplaba un bloque común y obligatorio, de 18 créditos, y un bloque de 
actividades electivas, de 6 créditos. Dentro de estos componentes curriculares obligatorios se encuentran seminarios de investigación ( 4 créditos), trabajos de comunicación científica ( 2 créditos) y tres disciplinas con formación integradora con un abordaje del contexto regional: Escenarios de política pública de educación en América Latina (4 créditos), bases epistemológicas de la investigación en educación (4 créditos) y profesión docente en América latina (4 créditos). Se sugiere además la posibilidad que todos los alumnos realicen un intercambio doctoral en otro país del subcontinente por un periodo mínimo de seis meses (DLA, 2009, p. 23).

La expectativa era de que todas las IES participantes comenzaran la oferta del Doctorado Latinoamericano al mismo tiempo en sus respectivos países, lo que no pudo darse debido a las diferentes condiciones de los actores; pues confluyeron en la iniciativa países con sistemas de postgrado ya consolidados, como el Brasil y México, pero también Estados con poca tradición en la oferta del doctorado, como es el caso Honduras.

\section{HETEROGENEIDAD DE LOS ACTORES EN JUEGO}

Primeramente, es necesario esclarecer que la participación de la Unesco en el proyecto del DLA fue de forma temporal, con una función de articulador internacional, dado su prestigio y su capacidad de incentivar el envolvimiento de las instancias máximas de cada universidad participe, para que legitimen y apoyen plenamente la propuesta en construcción. En este sentido, su papel fue el de convocar la instituciones y coordinar de forma colegiada las reuniones en que se establecerían las directrices del trabajo interuniversitario y organizacional, apoyando también la búsqueda de recursos y la difusión de informaciones sobre el doctorado (DLA, 2009, p. 6, 7).

La Unesco acompaño efectivamente todo el proceso hasta el lanzamiento del programa, siendo el Grupo de Trabajo (GT) quien firma la redacción final del DLA compuesto por un representante de la Orelac, un representante del Iesalc, un profesor de la Universidad Pedagógica Experimental Libertador de Venezuela, uno dela Universidad Pedagógica Nacional do México, uno de la Universidad Pedagógica Nacional da Colombia e dos profesores de la Universidad Federal de Minas Gerais; contando también oportunamente la contribución de los representantes de las demás IES que firman el acuerdo. 
Es importante resaltar también la mediación de la Unesco para la incorporación de la Universidad Federal de Minas Gerais en la propuesta del doctorado. La UFMG inicialmente no estaba vinculada a la Red Kipus, que congrega en mayor parte universidades pedagógicas, que son instituciones superiores dedicadas exclusivamente a la formación de los profesionales de la educación. Consiste en un modelo de IES bastante común en diversos Estados de la América Latina y el Caribe, pero que no se encuentra en Brasil. A pesar de esto, este país pasa a ser identificado como un participante importante en la construcción del DLA por su experiencia en la oferta de programas de postgrado, siendo, en cuestión de números totales, el principal formador de doctores en ALyC.

Además el Brasil vivía un contexto favorable para la realización de este tipo de emprendimiento, con la disposición para la integración regional que se tenía en los gobiernos Lula da Silva. El proyecto coincidía, por tanto, con un interés latente de la administración pública brasilera, con una voluntad preexistente para desarrollar proyectos de cooperación a nivel del subcontinente.

De esta manera, después de que se consolido la idea de integrar este país a la propuesta del DLA, la invitación a la Universidade Federal de Minas Gerais fue hecha a través de la intermediación de la entonces directora del Iesalc, la brasilera Ana Lúcia Gazzola, ex rectora de la UFMG. A pesar de no haber participado de los dos primeros encuentros en que se idealizo el Doctorado Latinoamericano en Educación: Políticas públicas y profesión docente, esta institución ha sido un actor de gran relevancia en el proceso de construcción del programa, teniendo en cuenta su babitus particular, su capacidad desarrollado a lo largo de su trayectoria para estructurar un postgrado stricto sensu de nivel internacional. Frente a los demás participantes, la universidad brasileña tenía más atributos concretos para contribuir en la conformación del DLA, por su estructura física y administrativa, su cuerpo docente calificado, su experiencia en investigación, su programa de postgrado en educación ya consolidado y también por tratarse de una universidad pública gratuita, que podría venir a atraer y formar un conjunto de profesores latinoamericanos que en otros contextos no tendrían condiciones de obtener un grado de doctor.

La Universidade Federal de Minas Gerais fue la primera institución que ofreció el Doctorado Latinoamericano en Educación, con la apertura del proceso de selección de alumnos en el segundo semestre del 2010 y el inicio del curso en 2011. En las demás IES el programa 
solo vino a comenzar en los años siguientes - en 2012 en la Universidad Pedagógica Experimental Libertador de Venezuela, en la Universidad Pedagógica Nacional de Colombia y en la Universidad Pedagógica Nacional de México y en 2015 en la Universidad Pedagógica Nacional Francisco Morazán de Honduras, realizándose actualmente en un total de cinco sedes, incluido el Brasil. Al final de 2017, la evolución del DLA era bastante discrepante entre los polos: En la UFMG ya habían siete cohortes, en Venezuela y México dos y en la Universidad de Honduras apenas una, que, sin embargo cuenta con cuarenta alumnos, según informaciones de la coordinadora del programa de Postgrado en educación de la UFMG (PPGE/UFMG); ${ }^{4}$ mientras que en otras universidades la propuesta de formación aún no había sido totalmente implementada y se encontraba vinculada apenas a líneas de trabajo o investigación. Además es importante destacar la infraestructura y el cuadro docente de cada institución, que en muchos casos cuenta con bajo número de doctores lo que imponen ritmos diferentes al proceso.

Vale señalar que la Unesco se separó del proceso después de su lanzamiento, siendo instituida una Coordinación Académica Internacional, compuesta por un docente representante de cada institución sede, que es actualmente la instancia máxima de dirección académica del programa. Tal consejo directivo fue coordinado hasta noviembre de 2013 por el venezolano Pablo Ríos, asumiendo entonces la coordinación la profesora mexicana María Torres, que estuvo en el cargo hasta abril de 2017, cuando la brasileña Lívia Maria Fraga Vieira asumió la función. El grupo se articula principalmente por medios virtuales, tal como una rutina de reuniones por video conferencia y la realización más ocasional de encuentros presenciales (Caracas, en 2010; Belo Horizonte, en 2011, 2013 y 2015; México, en 2016). A pesar de la distancia, esas reuniones han constituido un espacio importante para compartir las experiencias y profundizar la cooperación entre las universidades.

Veamos cómo se ha dado la inserción del Doctorado latinoamericano en la Universidad Federal de Minas Gerais.

\section{LA IMPLEMENTACIÓN DEL DOCTORADO LATINOAMERICANO EN EDUCACIÓN EN LA UFMG}

El surgimiento del postgrado en educación en Brasil data de la década de 1960, cuando fue creada la maestría en educación de la Pontificia Universidad Católica do Rio de Janeiro. La expansión del área 
ocurriría en la siguiente década, impulsada por la estructuración del Sistema Nacional de Postgrado, y es en ese movimiento que la UFMG inicio en 1971 la oferta del curso de maestría en educación. Ya el doctorado solamente vendría a funcionar veinte años más tarde, a partir de 1991. Al lanzar la convocatoria para abrir la primera cohorte del DLA, el PPGE de la UFMG ya era considerado como uno de los mejores y más tradicionales del Brasil.

En 2010, de los 98 programas de postgrado en educación existentes en el país, apenas ocho eran considerados de excelencia internacional (notas 6 y 7), según la evaluación de la Capes, en la cual la Universidad Federal de Minas Gerais había obtenido el concepto máximo. ${ }^{5}$

El programa, en la perspectiva de los evaluadores ${ }^{6}$ presentaba un alto nivel de calidad, de producción y de desempeño compatible con los de los principales centros internacionales de investigación en el área de educación, con una importante inmersión en el exterior comprobada por los indicadores de publicación, de movilidad discente y docente y por los convenios establecidos con universidades extranjeras de reconocida calidad académica. Dentro de las interacciones en el ámbito de América Latina y el Caribe, se destaca la vinculación del PPGE/UFMG al consejo Latinoamericano de Ciencias Sociales (Clacso) y la Red Latinoamericana de Estudios Sobre Trabajo Docente (Red Estrado).

En medio de este contexto favorable, el DLA fue creado como una modalidad de curso en el interior del programa de Postgrado en Educación de la UFMG, junto con la maestría y el doctorado regular que ya eran ofrecidos. La idea inicial consistía en crear un programa independiente, sin embargo en este caso el DLA podría encontrar más dificultades de orden presupuestal, una vez que en el Brasil las becas de estudio y otros tipos de financiación privilegian los programas ya consolidados. La estrategia de incorporar el DLA al PPGE fue discutida con el entonces director de Relaciones Internacionales de la Capes, y discutida también con la pro rectoría de investigación y postgrado de la UFMG y con el colegiado del programa, hasta llegar a la conclusión de que esa forma sería la más efectiva para la implementación del nuevo proyecto.

En el ámbito del Colegiado del programa las discusiones fueron intensas, sobre todo en lo que respecta al espacio a ser ocupado por el DLA en la estructura del PPGE - si el sería instituido como una nueva modalidad de curso, o si le quedaría apenas el lugar de una nueva línea de investigación a ser creada dentro del doctorado que ya existía. 
Prevaleció la constitución del DLA como una modalidad especifica del curso. Se observaron ciertas resistencias que pueden ser atribuidas al hecho de ser un doctorado con América Latina, con países más pobres que el Brasil. Aún con el reconocimiento de la relevancia de la relación con Latinoamérica, de la conciencia de la pertenencia del Brasil a una región específica, a menudo la internacionalización con América Latina no es valorizada ni en la UFMG ni en las agencias de incentivo a la investigación.

Además de esa relativa resistencia y el poco valor que se le da a la cooperación Sur-Sur y de la disputa por recursos, otra cuestión a ser enfrentada fue el hecho de que el PPGE no contaba con un cuerpo docente formado con vocación latinoamericana y caribeña, de manera que la investigación de enfoque regional estaba circunscrita a pocos profesores. La resolución que define las normas del funcionamiento del DLA establecía, en el artículo $6^{\circ}$, que los docentes interesados en ofrecer cupos en esa modalidad deberían comprobar inserción en $\mathrm{ALyC}$, por medio de la realización de investigaciones, cooperaciones, participación en redes, contactos y/o experiencias académicas (UFMG/PPGE, 2013, n.p.).

Otro factor que restringía el vínculo de un número mayor de profesores está relacionado a la especificidad de los temas de investigación, que se esperaba estuviesen principalmente concentradas en el campo de las políticas públicas y de la profesión docente. De los aproximadamente 75 profesores autorizados para orientar doctorado, del cuadro del PPGE/UFMG al final de 2016, cerca de un tercio (26) había abierto cupos en las convocatorias específicas para el curso latinoamericano desde el lanzamiento de esa modalidad. Sin embargo, desde 2014, con la inclusión de nuevas líneas de investigación este escenario cambia, ampliándose con él también la orientación de las tesis, pero aunque se amplía el espectro de investigación, no se amplía significativamente el número de docentes.

Es posible verificar que a lo largo de los años al menos un nuevo profesor se integró al DLA a cada convocatoria. Al mismo tiempo se identifica que la mayoría de docentes no persiste en la oferta de cupos después de una primera experiencia. Apenas ocho profesores ya orientaron más de un alumno en este curso específico. Esta inclusión dispersa del cuerpo docente al DLA puede estar relacionada a la limitada conexión de los profesores con la investigación regional y con el tema de la profesión docente o aún con las especificidades de orden burocrática del curso. 
El DLA posee normas especiales de funcionamiento, relativas a las particularidades de un curso internacional e interinstitucional. Las disciplinas obligatorias no son las mismas impuestas a los alumnos del curso regular, se contemplan cuestiones relativas a la acogida de los estudiantes extranjeros - como hospedaje y obtención de visa - y la obligatoriedad de una estancia de investigación en otro país de la región, que ha sido impuesta para los alumnos brasileños (modalidad doctorado sándwich). A pesar de lo anterior, no existía, hasta recientemente, una secretaría exclusiva para el DLA. Solamente se estableció a partir de 2016, la asignación de una funcionaria para el dialogo con los estudiantes y docentes vinculados al programa, con el objetivo de cuidar de los diferentes aspectos administrativos relativos al DLA. ${ }^{7}$

\section{SELECCIÓN DE LOS ALUMNOS}

El proceso de selección para el Doctorado Latinoamericano en Educación PPGE/UFMG es realizado por convocatoria específica para el curso. La selección se da en dos etapas, ambas de carácter eliminatorio y clasificatorio. La primera consiste en la evaluación del proyecto de investigación, que puede estar escrito en portugués o español, y debe abordar temas educativos pertinentes a la realidad regional, específicamente aquellos relacionados a políticas públicas y profesión docente. En la segunda etapa, ocurre la evaluación de los currículos de los candidatos y la defensa oral de los proyectos, que puede darse a distancia, vía videoconferencia, con el fin de facilitar la participación de los concurrentes extranjeros.

Los candidatos brasileños, tienen como exigencia que los proyectos de investigación cumplan alguno de los siguientes requisitos: i) tratar de temas educativos, en contraste o comparación de la realidad de países latinoamericanos o ii) que estos temas sean, por lo menos, sobre un país de América Latina, excepto el Brasil (UFMG/PPGE, 2013, n.p.). De esta manera se promueve efectivamente la formación de doctores brasileños en educación en una perspectiva ampliada, más allá de la nacional.

La movilidad académica representa otra condición obligatoria para los brasileños, que deben permanecer en otro país de la región por un período mínimo de seis meses. Este requisito ha provocado críticas entre los aspirantes, una vez que el programa no garantiza becas o ayuda financiera para la realización del intercambio, siendo responsabilidad de cada alumno garantizar los medios para su estancia en el exterior. En 
las demás sedes del DLA esa cláusula ya fue flexibilizada, justamente por la imposibilidad de que las instituciones ofrezcan los recursos financieros necesarios para el apoyo a los estudiantes.

En el caso de los alumnos extranjeros matriculados en la UFMG, en ese curso específicamente, el reglamento establece la obligatoriedad de la residencia en Belo Horizonte por lo menos en el primer año. Para este caso se cuenta con una beca otorgada por una agencia de financiamiento nacional, reservada para esta finalidad por el PPGE a cada nueva convocatoria. También se espera que el grupo pueda reunirse en el Brasil en el último semestre del doctorado, para la conclusión de la tesis, pero sin garantía de apoyo financiero. De todas formas, las becas constituyen un elemento de gran importancia para aumentar el interés y la permanencia de los estudiantes internacionales y representa el compromiso de la UFMG con una internacionalización solidaria en el ámbito de América Latina y Caribe.

En relación a la cuestión lingüística, el curso se inspira en el principio de la reciprocidad, que posibilita que cada participante desarrolle su comunicación oral y escrita - desde trabajos de evaluación de las disciplinas e incluso la redacción de la tesis - tanto en el idioma portugués como en el español. En la misma premisa de proporcionalidad, se solicita de los brasileños la certificación en español como lengua adicional y de los extranjeros se exige dominio del portugués, que pueden ser comprobados, por medio de examen, en un plazo máximo de 24 meses después de la matrícula. Además se exige comprobar un segundo idioma adicional, pero en este caso los alumnos pueden escoger entre inglés, francés e italiano.

Al final del segundo semestre lectivo de 2018, el DLA acumulaba un total de 57 matrículas desde su primera oferta, siendo 28 de brasileños y 29 de extranjeros, segundo las nacionalidades indicadas en la Tabla 1. De estos, 28 ya habían obtenido su título de doctor y los demás se encontraban en cursos. 
TABLA 1. $N^{0}$ de nuevas matrículas en el Doctorado Latinoamericano en la UFMG por nacionalidad y por año, 2011-2018

\begin{tabular}{|r|c|c|c|c|c|c|c|c|c|}
\hline \multirow{2}{*}{ Nacionalidad } & \multicolumn{8}{|c|}{ No de nuevas matrículas por año $^{2}$} \\
\cline { 2 - 12 } & 2011 & 2012 & 2013 & 2014 & 2015 & 2016 & 2017 & 2018 & Total \\
\hline Brasil & 3 & 2 & 3 & 3 & 5 & 4 & 4 & 4 & 28 \\
\hline Argentina & 1 & 1 & 1 & 1 & 1 & - & 1 & - & 6 \\
\hline Chile & - & - & 1 & - & - & - & - & 1 & 2 \\
\hline Colombia & 1 & 1 & 2 & 2 & 1 & 2 & 1 & 2 & 12 \\
\hline Ecuador & 1 & 1 & 2 & - & - & - & - & - & 4 \\
\hline Perú & - & 1 & 1 & - & 1 & - & - & 1 & 4 \\
\hline Cuba & - & - & - & - & - & - & 1 & - & 1 \\
\hline Total & 6 & 6 & 10 & 6 & 8 & 6 & 7 & 8 & 57 \\
\hline
\end{tabular}

Fuente: Elaboración propia con datos fornecidos por el Programa de Postgrado en Educación de la Universidad Federal de Minas Gerais.

A pesar que las convocatorias no especifican el número de cupos destinados a estudiantes brasileños y a estudiantes de otros países latinoamericanos, se observa un equilibrio entre la participación de nativos y extranjeros. Por otro lado, se verifica un desnivel en la distribución de las matrículas internacionales, siendo aproximadamente el $41 \%$ de los cupos ocupados por colombianos. El bajo número de países participantes es otro factor curioso, una vez que el curso no se dirige apenas a los estados relacionados en el Acuerdo de Cooperación del DLA, pero sí para toda la región. Vale la pena destacar que la divulgación de las convocatorias de selección ocurre principalmente por medios virtuales, por los fórums en los que el PPGE/UFMG participa, como Clacso, la Red Estrado u la Red Kipus. La ANPEd - Asociación de postgrado y pesquisa en educación de Brasil también colabora en la divulgación de las convocatorias de selección. El consejo académico internacional reconoce la necesidad de ampliar la comunicación en otros espacios, con el objetivo de asegurar candidaturas suficientes para una buena selección. La construcción de un website oficial sobre el Doctorado Latinoamericano, por ejemplo, donde constarían informaciones de todas las sedes, sus convocatorias y producciones, es una demanda que aparece con persistencia en las relatorías del Consejo. La UPN de México había asumido esa responsabilidad en una reunión en marzo de 2010, en Caracas, más el desarrollo de la plataforma virtual permanece pendiente (DLA, 2010, p. 4; DLA, 2013, n.p.). 
En relación al perfil de los 57 matriculados, los cupos están igualmente distribuidas entre hombres (29) y mujeres (28), contrariando los indicadores regionales que apuntan que aproximadamente $75 \%$ de los profesores de América Latina y el Caribe, uno de los públicos - albo del DLA, son del sexo femenino. ${ }^{8}$ Es necesario considerar que en lo que tiene que ver al acceso a postgrado en el subcontinente la proporción por género tiende mismo a ser equilibrada. En el caso del Brasil, según los datos generales de la Capes, 53,2\% de los doctorandos matriculados en 2015 eran del sexo femenino.

En cuanto al currículo académico y profesional de los alumnos, de manera general, el curso ha recibido alumnos internacionales bastante cualificados, en cara a la demanda de los Estados vecinos por una formación doctoral. Muchos de estos extranjeros ya eran profesores universitarios en sus países con larga experiencia. De hecho entre los exalumnos se encuentran exdirector/a del escritorio regional de la Unesco, profesores universitarios del Ecuador, Argentina, Colombia y Perú.

Sobre los alumnos brasileños, una de las dificultades se encuentra en la escasa comprensión de América Latina, sobre las diferencias entre los países, la política, lo que sucede en estos países, las costumbres, el lenguaje. En ese contexto, el Doctorado Latinoamericano en Educación de la UFMG tiene un papel muy importante para promover el conocimiento de los investigadores brasileños sobre la realidad de la ALyC y el gran desafío de ayudar a crear un sentido de pertenencia regional.

\section{EDUCACIÓN COMPARADA}

Teniendo como referencia la estructura curricular común definida entre las IES participantes del DLA, el PPGE/UFMG complementa la formación de los estudiantes con electivas que son ofrecida en el programa de postgrado así como mini-cursos dirigidos a temas específicos de interés de la comunidad académica, algunos de esos cursos han sido: Escenarios de Políticas Públicas en América Latina, ampliando las discusiones de la disciplina obligatoria y convocando a profesores y estudiantes de los otros programas doctorales; Los Docentes y el Plan Nacional de Educación; El Caso de la Docencia en América Latina: Políticas, Evaluación y Formación; Investigación en Educación Comparada: Elementos Teóricos y Metodológicas. 
Los contenidos programáticos de las disciplinas han venido siendo construidos desde una perspectiva latinoamericana, trayendo autores y teorías que ayuden a pensar la región. Partiendo del presupuesto de que pensando la educación en América Latina: políticas, sistemas educativos, formación de educadores, derechos sociales, cultura etc. a partir del diálogo y reconocimiento del otro es posible profundizar las reflexiones sobra la educación en cada nación, ya sea en el diseño de investigaciones que permitan comprender la diversidad de experiencias nacionales o desde el contraste y comparación de las mismas. De ahí que una de las propuestas de este doctorado sea que sus estudiantes usen referentes de educación comparada en sus trabajos de investigación. El DLA de Brasil, como ya fue dicho, invita a los no brasileños que entran al programa al estudio de experiencias de los países de los que provienen; y a los brasileños a ver de forma comparada realidades del Brasil y otro u otros países de América Latina. Pero más allá de esta especificidad metodológica en la orientación de las tesis, el DLA se inscribe en la educación comparada en la misma lógica de su estructura. Pues posibilita el encuentro académico de personas, contextos, lenguas y nacionalidades diferentes, para pensar la educación en la región, provocando el reconocimiento de otros sistemas y políticas educativas que pueden ser usados como referentes para entender la educación en América Latina.

Desde el conocimiento y diálogo con el otro es posible comprender y entender la educación de las diferentes naciones, los sistemas globales e internacionales y con esto, tener un referente complejo para entender los rumbos educativos locales. Esta premisa es la que se defiende en las nuevas tendencias de la educación comparada que se preocupan por entender la educación desde el reconocimiento de los múltiplos actores y propósitos - económicos, culturales y políticos - que han influenciado el desarrollo de los diferentes sistemas y políticas educativos (ARNOVE, 2003; BRAY, 2010). Siendo así, al preguntarnos por la educación en América Latina, es importante no solo el reconocimiento del propio sistema de interés, si no su análisis cruzado con experiencias similares, para entender las singularidades y los factores comunes que puedan incidir en la realidad de estudio. Desafío que ha encontrado en la propuesta educativa e investigativa del DLA un escenario viable para su ejecución. 


\section{MÉRITOS Y DESAFÍOS DEL DLA DE LA UMFG EN LA PERSPECTIVA DE LOS EGRESADOS}

Al terminar el primer semestre de 2018, cinco cohortes habían concluido el Doctorado Latinoamericano en Educación: Políticas Públicas y Profesión Docente ofrecido por PPGE/UFMG. En total veinte ocho sujetos presentaron sus teses: once brasileños, seis colombianos, cuatro ecuatorianos, cuatro argentinos, dos peruanos y un chileno. Nuestro universo de investigación conto con la participación de las primeras cuatro cohortes que habían concluido su doctorado hasta el segundo semestre de 2016, periodo de levantamiento de datos para este estudio, veintidós personas siendo ocho brasileños, cuatro colombianos, cuatro ecuatorianos, tres argentinos, dos peruanos e un chileno. El término de consentimiento libre y esclarecido, juntamente con el cuestionario elaborado, fueran enviados en versiones en español y en portugués a todos, a través de e-mails individuales, obteniendo un índice general de respuesta de 77\%. Dentro de los brasileños, el índice alcanzo 87,5\% con siete participantes; y entre los extranjeros la participación fue del $71,4 \%$ con diez encuestados.

El cuestionario, se puso disponible en la plataforma GoogleForms y presentaba preguntas abiertas y cerradas sobre sexo, edad, formación, conocimiento previo sobre el Brasil y sobre la UFMG, la motivación para cursar el DLA, financiación de la permanencia en el exterior o en el país, según el caso, relevancia del curso, entre otros asuntos relacionados a la experiencia internacional que serán discutidos a continuación.

Con relación al género de los encuestados, en el grupo de brasileños habían dos personas del sexo femenino y cinco del masculino; entre los extranjeros eran cinco femenino y cinco masculino, de modo que el número general de participantes del sexo masculino (10) es poco superior del femenino (07), así como en el universo de todos los alumnos egresados del DLA de la UFMG.

En cuanto a la edad media de los titulados de la muestra, al final del 2016 correspondía a 47 años, siendo que los dos alumnos mayores tenían 65 años. Los estudiantes internacionales representan un grupo más longevo, con $50 \%$ de sujetos con edad superior a los 50 años y la media está situada en 52 años. Entre los brasileños, la media era de 41 años y el encuestado con más edad tenía 50 años en el momento de la investigación.

Sobre el lugar de residencia actual, todos los nativos informaron que continúan viviendo en el Brasil. Entre los extranjeros, siete de ellos 
se encuentran en sus países de origen y tres se encuentran en otro país latinoamericano diferente de su nacionalidad: dos ecuatorianos que mantenían su residencia en Perú y un colombiano que había permanecido en Belo Horizonte Brasil después de su titulación de doctorado.

En relación a su trayectoria académica anterior al ingreso en el DLA, entre los siete brasileños, cinco habían completado su carrera de grado en instituciones federales (siendo tres de ellas en la UFMG), un en IES estadual y un en IES privada. En cuanto al curso de Maestría, todos la realizaron en el área de educación en universidades públicas federales (cinco en la UFMG y dos en otras IES). Los siete sujetos afirman haber tenido acceso a la convocatoria del curso latinoamericano a través de los canales de comunicación de la UFMG, como la página web de la Facultad de Educación, a través de mensaje enviado a la base de datos de exalumnos o por indicación de colegas y profesores. Sobre la motivación para participar en el DLA, seis de los encuestados señalaron que ya tenían un interés por profundizar la investigación sobre el tema de educación con perspectiva regional y apenas uno afirmo que no se trató de una elección y sí de una oportunidad, habiendo adaptado su proyecto original para una perspectiva comparada para que pudiera presentarse al curso.

En el caso de la formación de los extranjeros, la mitad de ellos informa haber sido responsable por la propia formación de grado y $80 \%$ por costear su propia maestría. ${ }^{10}$ En lo que tiene a ver con el lugar de realización del pregrado, nueve cursaron en sus estados de origen y uno no respondió a la pregunta. En cuanto a los países de realización de la maestría, tres participantes presentan formación internacional: dos en el Brasil (uno en la Universidade Federal do Rio Grande do Sul y otro en la Universidade Estadual de Campinas, declarando los dos sujetos, que realizaron sus cursos gratuitamente) y una tercera persona que acumulaba dos maestrías, siendo una titulación en su país de nacionalidad y la segunda en Bolivia.

Dentro de los egresados extranjeros que respondieron al cuestionario, mitad nunca había escuchado hablar de la UFMG antes de la convocatoria del DLA. Pero la mayoría había visitado el Brasil previamente sea por motivos profesionales o académicos (3), por turismo (2) o por ambos motivos (1). Cuestionados sobre los canales de comunicación a través de los cuales supieron de la convocatoria de selección, cinco sujetos contaron que se enteraron por medio de relaciones interpersonales con colegas y docentes; dos personas indicaron que se enteraron por medio de redes regionales de la investigación sobre la educación (citando la Red Estrado 
y la Infeies - Red Interuniversitaria Internacional de Estudios e Investigaciones Interdisciplinarias e Infancia e Instituciones); una persona tuvo acceso a la convocatoria a través del consulado del Brasil en su país; otra indico la web, sin determinar la página; y una última estuvo directamente involucrada en la creación del DLA, pues era representante de la Unesco en el GT que estructuro el programa.

Acerca de los motivos para que no realicen el doctoramiento en sus países de origen, las principales respuestas fueron debido al alto costo financiero de esa modalidad stricto sensu (3) y por el hecho de que en sus países no ofrecen o cuentan con programas de doctorado en educación (5); Hubo incluso un sujeto que señalo que recurrió al Brasil por la calidad del sistema nacional de educación superior y otro que informo que tenía razones laborales, personales y políticas para no continuar los estudios en su país. De los diez encuestados internacionales, tres nunca habían pensado anteriormente en realizar un doctorado en el exterior; otros tres nunca habían pensado en cursarlo en algún otro país vecino; y cuatro ya habían imaginado dar continuidad a su trayectoria académica en un país de otro continente.

Se concluyen a partir del perfil de los ex-alumnos, que la oferta del DLA de la UFMG responde a una fuerte demanda para la formación en alto nivel de los profesionales de la educación con una perspectiva regional, así como era esperado cuando se estructuro el programa. La iniciativa fue especialmente atractiva para los alumnos internacionales, una vez que la mayoría de ellos ya estaban inseridos en actividades del área y no habían dado continuidad a su trayectoria de formación por la dificultad de hacerlo en sus países de origen - sea por la limitación de la oferta del doctorado o por los costos vinculados dentro de otras razones menos frecuentes.

Es importante destacar que los extranjeros entrevistados tuvieron garantizada beca de estudios para su permanencia en el Brasil, en el menor de los casos por el tiempo regularmente exigido (primer año del curso); mientras que los brasileños tuvieron que buscar individualmente los recursos para el financiamiento del intercambio en el exterior, disputando con los demás alumnos de pos graduación. De los siete nativos, seis obtuvieron éxito en la obtención de becas para su intercambio, y uno viajo con fondos propios. Ellos argumentan que una vez que la movilidad es una obligatoriedad curricular, debería garantizarse el aporte financiero por parte del PPGE/UFMG, pues el tratamiento diferenciado para extranjeros puede venir a construir un punto de tensión y división dentro de la categoría discente. 
A parte de eso, desde 2015 las agencias brasileñas de fomento han restringido la concesión de becas de intercambio internacional, disminuyendo el presupuesto y pasando a priorizar actividades en países desarrollados, de manera que el cumplimiento de la práctica internacional compulsoria se torna un desafío aún mayor a ser superado en el ámbito del DLA. Vale destacar que entre los brasileños encuestados, que se titularon entre los años lectivos de 2013 y de 2016, cuatro alumnos cumplieron el tiempo mínimo reglamentar de la practica en el exterior. Sin embargo los otros tres realizaron el intercambio por apenas cuatro meses y aun así tuvieron la actividad convalidada por el Colegiado del PPGE/UFMG.

Veamos, por fin, cual es la evaluación que los alumnos hacen de la experiencia de formación académica fuera de sus Estados de origen y de la pertinencia del DLA como un todo. Los estudiantes brasileños tuvieron como destino Argentina (3), Uruguay (2), Chile (1), México (1) y Colombia (1), siendo que un mismo alumno realizo el intercambio internacional de forma combinada en dos países (Argentina y Uruguay). Cuanto las universidades de acogida, al contrario de lo que se propone en el proyecto del DLA, la mayoría de los alumnos no se dirigió a las otras sedes del programa. En primer lugar, Argentina y Uruguay no integran la propuesta del DLA. Al mismo tiempo que Chile, a pesar de no haber constituido una sede del DLA, es interlocutor en la creación del curso a través de la adhesión de cuatro de sus IES; lamentablemente ninguna de las instituciones de este país fue escogida por los estudiantes para el intercambio. Solamente en Colombia y en México el intercambio se hizo en los polos del DLA, siendo que el doctorando que realizo sus actividades en la Ciudad de México colecto datos también en otras instituciones diferentes de la UPN.

Cuatro alumnos ya habían visitado anteriormente los países objeto de estudio, por motivos profesionales y/o académicos. Al mismo tiempo, igual número de entrevistados nunca había escuchado hablar de la IES escogida. Algunos de ellos relataron en las encuestas que el convenio institucional que viabilizo en intercambio era "frágil" y que "este debería ser intermediado por la coordinación del DLA", con el fin de que las actividades internacionales pudieran ser más alineadas de tal manera que se pueda fortalecer el proceso de formación y la cooperación académica (EgresadoDLA_brasileño1 e EgresadoDLA_ brasileño2). Sobre este asunto dos egresados brasileños manifestaron haber tenido mucha dificultad para resolver cuestiones administrativas en las IES receptoras, tales como registro, acceso a las bibliotecas e inserción en el cotidiano universitario; en contra posición la mayoría (5) tuvo poca o ninguna dificultad en estos asuntos. 
A pesar de los inconvenientes relatados, todos los participantes de la investigación calificaran como muy positiva la experiencia del intercambio doctoral en el exterior. Ellos señalaron que esta experiencia les otorgo más madurez y autonomía personal, por los desafíos inherentes al proceso, como la necesidad de adaptarse en medio a los otros hábitos y costumbres, construir nuevos lazos de amistad y desarrollar el autoconocimiento. Es posible afirmar también, a partir de los relatos, que la movilidad contribuyo a la aproximación y asimilación de los titulados de una identidad latinoamericana:

[Fue una experiencia] importante para percibir nuestra lejanía/ausencia de un sentido de pertenencia [regional]. (EgresadoDLA_brasileño1)

Solamente cuando pasamos a vivir en otra realidad latinoamericana es posible reconocer cuanto nuestro país está alejado de una red más amplia. (EgresadoDLA_ brasileño2).

Los aprendizajes, amistades e intercambios generados a partir de la vivencia cotidiana con profesores, estudiantes y nativos ampliaron mi repertorio cultural y, necesariamente, ampliaron mi sensibilidad para con la historia y temas específicos de esos sujetos (EgresadoDLA_brasileño3).

Pienso que fue una valiosa oportunidad para conocer mejor el pueblo de un país vecino, influyente, y poner en jaque muchas impresiones equivocadas que poseía sobre ellos (EgresadoDLA_brasileño4).

Ese sentido de pertenencia es fundamental para el desarrollo de proyectos futuros de integración latinoamericana y caribeña, que tienen como pauta el respeto por la diversidad de los países, sus saberes y conocimientos. Responde, por tanto, a los objetivos del DLA de crear una masa crítica inspirada por principios de solidaridad y comprometida con las cuestiones regionales (DLA, 2009, p. 10).

Los egresados brasileños valorizan la realización de los estudios comparados, que pudieron "profundizarse" en el intercambio, por ser una "experiencia riquísima de investigación y trabajo de campo" (EgresadoDLA_brasileño1) lo que queda evidente en el testimonio de otro de los participantes: "Tuve la oportunidad de descubrir detalles de otro sistema educativo público [...]. Conviví con una organización universitaria diferente de aquella en la que estaba matriculado como doctorando y de aquella en la que soy profesor efectivo. Visite escuelas [de la capital y del interior] del país [donde realice el intercambio]" (EgresadoDLA_brasileño6). En este contexto, enfatizan que el intercambio "favoreció una lectura crítica de la realidad educacional en América Latina", constituyendo una "oportunidad de comprensión del fenómeno educativo de manera más incluyente y plural" (EgresadoDLA_brasileño3 e EgresadoDLA_brasileño5). 
Otro mérito de la movilidad estudiantil, según los sujetos que respondieron la investigación consiste en profundizar los lazos académicos. Ellos relatan:

El Doctorado Latinoamericano es uno de los programas más importantes en educación en América Latina. El impacto de su propuesta en red está visiblemente siendo construido. En cuanto egresado, actualmente doy continuidad a mi investigación de forma institucional dentro de la IES donde actuó (EgresadoDLA_ brasileño2).

La experiencia de la movilidad favoreció el establecimiento de intercambio de investigación. Hoy, como profesora [de educación superior], he buscado establecer proyectos con colegas del país en el cual hice el intercambio (EgresadoDLA_ brasileño4).

Me acerqué a personas que gestionan la política pública estudiada en el Doctorado [latinoamericano] (EgresadoDLA_brasileño6).

El DLA presenta, de esa manera, un gran potencial para los desarrollos de convenios internacionales productivos y duraderos. Después de la conclusión del curso, tres brasileños ya habían regresado a los países donde realizaron el intercambio por motivos académicos y/o profesionales y todos ellos deseaban regresar nuevamente a ese destino en un futuro próximo (en un periodo de dos años), sea para el desarrollo de actividades universitarias (6) sea para hacer turismo (1).

La opinión de los egresados extranjeros del DLA del PPGE/ UFMG también se mostró bastante positiva. En el caso de los alumnos que ven de otros estados latinoamericanos, se observa que la estadía en el Brasil fue bastante superior a la exigencia mínima de un año, con permanencia media de 27 meses. Se destaca que de los diez encuestados, 3 residían en el país durante los 4 años de curso y otros 2 doctorandos durante 3 años. Solamente dos sujetos estuvieron en Belo Horizonte en tiempo inferior al establecido - uno solamente por seis meses y el otro por ocho meses.

Sobre la adaptación de los alumnos en Brasil, ocho personas respondieron haber tenido poca o ninguna dificultad para comunicarse en portugués. Seis extranjeros afirman haber tenido poca o ninguna dificultad en lo que tiene que ver con los procesos de secretaria de la universidad, mientras que cuatro informaron un grado moderado de dificultad. Uno de esos sujetos resalto la dificultad de comunicación que se generó cuando ya no estaba en el país y necesitaba manifestar sus dudas vía correo electrónico, sin obtener una respuesta en un plazo oportuno. En relación a las cuestiones referentes a la instalación en Belo Horizonte (visa, habitad, etc.), seis personas encontraron dificultad moderada o grande, mientras que las otras cuatro tuvieron poco o 
ninguna dificultad. Continuando con las cuestiones de hospedaje, es importante resaltar que la Dirección de Relaciones Internacionales de UFMG gestiono una cuota en la Moradia Universitaria. A pesar de esto, como todos los apartamentos son compartidos - generalmente con alumnos de pregrado - la mayoría de los doctorandos del programa han optado por buscar otros espacios con mayor privacidad y mejores condiciones de producción de conocimiento. ${ }^{11}$

Sobre las relaciones interpersonales en el ámbito del PPGE, el conjunto de los diez egresados que respondieron la investigación evaluaron como buena o excelente la interacción tanto con los demás alumnos brasileños como con los orientadores. Ellos destacan la creación y el fortalecimiento de lazos de amistad con los colegas de curso y también la comprensión y los convenios establecidos con los orientadores. Y, si por un lado, consiguieron estar inmersos en la cultura brasilera, destacan también que pudieron contribuir "para fortalecer el espíritu latinoamericano" y que pudieron sentirse como "embajadores de sus países", con la oportunidad de desmitificar visiones estereotipadas sobre sus estados (EgresadoDLA_extranjero1, EgresadoDLA_extranjero2).

La formación en el Brasil fue clasificada como muy relevante por todos los titulados de otros países del subcontinente, que señalaron algunas de las contribuciones del DLA:

[El doctorado latino-americano posibilita un] intercambio académico enriquecedor para el desarrollo de saberes latinoamericanos. (EgresadoDLA_extranjero3)

Me permitió incorporar otros enfoques teóricos, actualizar debates y perspectivas teórico-metodológicas en el campo de la política educativa. Valoro fundamentalmente sus aportes en el tratamiento de la cuestión latinoamericana y la posibilidad de volver a trabajar con estudios comparados. Permite 'abrir las miradas', triangular y comparar desarrollos teóricos y metodológicos y compartir ámbitos académicos solidarios. (EgresadoDLA_extranjero4)

El doctorado latinoamericano fue una excelente experiencia profesional de aprendizaje, intercambio, fortalecimiento de relaciones profesionales y de oportunidad para producir conocimiento. (EgresadoDLA_extranjero5)

Mi nivel para dar clases, para presentar ideas [después de la realización del doctorado] es muy diferente y considero mucho mejor. (EgresadoDLA_extranjero6)

Los participantes extranjeros también destacan la UFMG como una universidad de nivel internacional, con programas de alta calidad académica. Señalaron la importancia de poder participar, durante su permanencia en Brasil, de importantes congresos del área de educación (haciendo mención a la Reunión de la Asociación 
Nacional de Postgrado e Investigación en Educación, Anped y el Encuentro Internacional de la Red Estrado), con la posibilidad de apoyo financiero del PPGE para los viajes en el que hubiera presentación de trabajo académico.

Mitad de las personas no habían retornado al Brasil después de la obtención del título de doctor, otros cuatro habían regresado a visitar el país por motivos profesionales y/o académicos y uno de los egresados extranjeros continuaba viviendo en Belo Horizonte en el momento de la aplicación de la encuesta, como profesor substituto de una IES pública. Sin embargo, supimos que en 2017 él había retornado a su país de origen, también como profesor universitario.

En cuanto a las opciones de empleo después de la realización del DLA, pudo verificarse que todos los participantes de la investigación - brasileños y extranjeros - se encontraban trabajando en educación, la mayoría (15) como docente, uno como director de una institución de educación superior y uno en una función de director de un organismo internacional vinculado a educación (Unesco).

El Doctorado en Educación: Políticas Públicas y Profesión Docente ha constituido un espacio privilegiado para discutir y generar conocimiento sobre la América Latina y el Caribe. Este programa ha contribuido, por un lado, para afinar el sentido de pertenencia de los brasileños a una cultura regional. Por otro lado, contribuye en la formación de cuadros docentes de alta calidad para países extranjeros, además de fortalecer alianzas que podrán resultar en proyectos y políticas públicas para mejorar la educación. Hay, a pesar de esto, una serie de desafíos para ser superados para conseguir el empoderamiento del DLA en la estructura del PPGE/UFMG, siendo necesaria una estructura administrativa adecuada para la gestión del curso, la ampliación del interés de los estudiantes en un escenario que, conforme lo ya señalado es de recortes en el presupuesto público para las actividades sociales.

Otra cuestión fundamental dice respecto al fortalecimiento de la alianza con las instituciones de educación superior participes del DLA, con la creación de medios que favorecen el intercambio de los discentes y docentes de las universidades sede. Hasta la fecha, son bastante dispersas las oportunidades de formación conjunta de los alumnos de los diferentes polos. En el diseño del programa había una propuesta de ofrecerse disciplinas comunes a distancia y de lanzar publicaciones que garantizaran la circulación del conocimiento producido en el ámbito de DLA. Lamentablemente ninguna de estas actividades se concretizo. Hubo apenas un primero encuentro de estudiantes del Doctorado latinoamericano, realizado en Belo Horizonte en marzo de 2015, 
envolviendo alumnos de Brasil, Venezuela, México y Colombia. A pesar de la actividad haber sido evaluada por posibilitar el intercambio de experiencias de investigación entre las sedes, canales de interlocución permanente aún no fueron criados.

\section{PROTAGONISMO DE LOS DOCTORANDOS}

Como se ha presentado en este documento la puesta en práctica del DLA en Brasil y su constitución ha dependido de un esfuerzo conjunto entre todas las personas que hacen parte de este programa. El DLA es un programa de gran importancia pero que por su perspectiva significa también un campo de disputa dentro y fuera de la universidad. La inclusión de nuevos discursos, de personas y lugares que cuestionan y amplían los referentes que tradicionalmente han acompañado la formación universitaria y la investigación en Brasil se colocan como grandes desafíos que exigen un esfuerzo de transformación y cambio para su instauración. Administrativos, técnicos, profesores y alumnos han participado de la construcción y consolidación de este proyecto educativo dentro del contexto académico de postgrado brasileño.

Los principales retos están relacionados con la necesidad de conquistar un lugar de reconocimiento político y un estatus académico dentro de la universidad que garantice las condiciones ideales para posibilitar los diálogos y discusiones pertinentes que conduzcan al estudio de la educación en América Latina. Los esfuerzos emprendidos han incluido el dialogo entre profesores y alumnos para la diversificación de autores y temáticas que componen los contenidos programáticos de las disciplinas; con el objetivo de conocer autores regionales, estrategias territoriales y estudios o pensamientos que dialoguen con la realidad de la región; la búsqueda y abertura de espacios de aprendizaje e intercambio académico para dar a conocer los trabajos que se desarrollan en el DLA y para conocer el estado de los estudios en educación y políticas públicas en la región; el intercambio cultural que permitan un contacto más cercano de las personas de la UFMG con América Latina; la consolidación de alianzas con grupos de investigación para ampliar el alcance, los recursos y las personas en el desarrollo de las diferentes actividades; la garantía de un esfuerzo adicional para garantizar tesis de calidad que reflejen las discusiones emprendidas.

Al primer semestre de 2018 se contaba con 28 teses concluidas dentro del programa doctoral, en un amplio espectro de temáticas, 
líneas y metodologías de investigación. Entre la diversidad de trabajos de tesis podemos reconocer algunas temáticas predominantes: la mayoría de las investigaciones reflexionan sobre el análisis o evaluación de políticas públicas o programas educativos (7 teses), la formación docente (5 teses), el trabajo y la profesión docente (5 teses), modelos y programas educativos específicos (3), y otros en menos medida abordan temáticas como universidad pública (1), enseñanza (2) o psicología (1). Todos estos trabajos aportando a la comprensión de la realidad de la educación y los desafíos que se les colocan a los educadores y responsables por la educación en América Latina.

El DLA cumpliendo con su objetivo de posibilitar espacios para ampliar el intercambio entre los países de ALyC ha organizado y acompañado algunos eventos académicos y culturales tales como: i) "Primer Encuentro Internacional de Estudiantes del Doctorado Latinoamericano en Educación: Políticas Públicas y Profesión Docente", Universidad Federal de Minas Gerais, Belo Horizonte, nos días 26-27-28 de marzo de 2015. Evento que reunió alumnos y profesores de los diferentes países que hacen parte del DLA. En esta oportunidad además se dio la primera Asamblea internacional de Estudiantes del DLA, del cual surgió un documento conocido como la "Carta de Belo Horizonte" con algunas reivindicaciones de los estudiantes del programa; Documento que presento al comité internacional del DLA las inquietudes, peticiones y compromisos de los estudiantes de las diferentes sedes, orientados a fortalecer y potencializar el proyecto de DLA en la región. ii) Seminario de Tesis del Doctorado Latino americano en Educación, evento que comenzó en 2014 y llego a su tercera versión en 2017. Este evento es un espacio institucionalizado en el que se presentan las investigaciones concluidas y aquellas que aún se encuentran en desarrollo, para compartir e intercambiar ideas que potencialicen la reflexión y los análisis sobre la educación en América Latina. Este seminario cuenta con transmisión on-line, estando disponible para las Universidades de la red, así como para curiosos e interesados en estas temáticas. iii) "Ciclo de Cine" espacio en el que se propone, a partir del cine, conocer y reflexionar sobre diferentes características educativas de la región. Las temáticas discutidas han sido Educación y Democracia en América Latina y Educación y Cultura política en América latina-, el evento consiste en la exhibición de películas de diferentes países, a la fecha Brasil, Colombia, Perú, Argentina y Chile, seguido de comentarios de los estudiantes oriundos y debate con los asistentes. A partir del final del 2018 fue iniciado un convenio del DLA con la sala de Cine Belas Artes de Belo Horizonte para realizar una "Sesión Latina 
de Cine" a cada dos meses, abierta a los estudiantes de la UFMG y docentes y estudiantes de las redes públicas de educación básica, con un debate al final de cada exhibición. iv) Presencia en eventos académicos, políticos y estudiantiles con la participación de diferentes especialistas en temáticas que profundizaban las discusiones dentro del DLA y que invitaban a la comunidad académica de la UFMG y otras universidades a reflexionar sobre los temas que son de interés para la educación en América Latina.

Esta construcción conjunta ha posibilitado estrechar relaciones de pertenencia y colaboración internacional. El DLA de Brasil se ha constituido como una referencia a nivel nacional e internacional, y aunque aún deben seguirse sumando esfuerzos para alcanzar el potencial de este programa, hoy ya se pueden ver los resultados y el efecto de las discusiones en los discursos de egresados, profesores y alumnos que hoy contribuyen al desarrollo de la educación en América Latina.

\section{CONSIDERACIONES FINALES}

El DLA corresponde, en nuestro análisis, un modelo alternativo e innovador de internacionalización en el contexto de los programas de postgrado de excelencia. De manera general, el curso ha logrado éxito en el atendimiento a una demanda latente en la región para la titulación en alto nivel de los profesionales en educación. Los egresos, nativos y extranjeros, clasificaron como muy relevante la formación propiciada durante la trayectoria en el DLA. Ellos destacaron las contribuciones del punto de vista instrumental, por el perfeccionamiento de las habilidades de producción de conocimiento relativo a la cuestión de las políticas públicas y de profesión docente; las contribuciones desde el punto de vista de las relaciones establecidas, resaltando los lazos de amistad y profesionales que podrán impulsar proyectos colaborativos en el futuro; y, aún los beneficios del punto de vista cultural, una vez que el curso ha inspirado el sentido de pertenencia a América Latina y el Caribe.

La sustentabilidad del Doctorado latinoamericano, no obstante, dependerá de cómo las relaciones de poder van a establecerse entre los agentes internos del PPGE/UFMG, pero también va a depender de la coyuntura política nacional, que puede inviabilizar o perjudicar fuertemente el progreso del curso, con la restricción presupuestal y una política de internacionalización orientada para los principales centros mundiales de producción de conocimiento. 
Por otro lado, el DLA ha propiciado ampliar las relaciones con universidades latinoamericanas e incluso europeas por medio de proyectos de investigación sobre los temas de la región coordinados por profesores del PPGE/UFMG. En 2018, comienzan a discutirse también experiencias de Co-tutelas, y hubo la inserción del DLA en los proyectos y acciones desarrollados bajo la convocatoria de la Capes/ PrInt, contemplado por la UFMG, lo que amplía las perspectivas para los años siguientes.

Al fin, nos parece necesaria la sistematización y divulgación de la producción académica de los estudiantes y docentes del DLA desde la perspectiva de poder evidenciar las contribuciones que este programa y sus alumnos han hecho para el análisis de las políticas educativas y a la profesión docente en la región. Esta puede ser una medida importante para subsidiar la elaboración de políticas públicas y la organización de los actores docentes/discentes/sindicalistas en la defensa e implementación de políticas que tengan por objetivo la inclusión y democratización, como contrapunto a las políticas conservadoras y neoliberales presentes en las reformas de la agenda educativa global.

\section{REFERENCIAS}

ARNOVE, Robert F. Reframing comparative education. In: ARNOVE, R. F. and TORRES, C. A. (Orgs.). Comparative education: the dialectic of the global and the local. Lanham: Rowman \& Littlefield, 2003, p. 1-23.

BRAY, Mark. Actores y propósitos en educación comparada. In: BRAY, M., ADAMSON, B. y MASON, M. (Orgs). Educación comparada: enfoques y métodos. Buenos Aires: Granica, 2010.

CAPES. Coordenação de Aperfeiçoamento de Pessoal de Nível Superior. Plano Nacional de Pós-Graduação 2011-2020. Brasília: Capes, 2010.

CARTA DE SANTIAGO. Memoria técnica del Seminario Latinoamericano de Universidades Pedagógicas "El Rol de las Universidades Pedagógicas en las Formación Docente: Experiencias y Desafíos”. Chile, p. 136-138, 2003.

DLA. Doctorado Latinoamericano en Educación. Programa de Doctorado Latinoamericano en Educación: políticas públicas e profissión docente. Caracas: julio de 2009. Documento impreso.

Relatoría reunión Doctorado LatinoAmericano: Taller Caracas. Caracas: marzo de 2010. Documento impreso.

Memoria de la reunión del Consejo de Coordinación Académica Internacional. Belo Horizonte: noviembre de 2013. Documento impreso.

OCDE. Organização para a Cooperação e Desenvolvimento Econômico. Education at a Glance 2016 - OECD Indicators. Paris: OECD Publishing, 2016. 
SOUZA, Juliana F. Itinerários da internacionalização da educação superior brasileira no âmbito da América Latina e Caribe. Tese de doutorado em Educação. Belo Horizonte: UFMG/FaE, 2018.

UFMG/PPGE. Programa de Pós-graduação em Educação da Universidade Federal de Minas Gerais. Resolução $\mathbf{n}^{\mathbf{0}}$ 3, de 18 de março de 2013. Dá nova redação à Resolução 02/2012 que define normas para o funcionamento do Doutorado Latino-Americano. Documento impreso.

UFMG/PPGE. Programa de Pós-graduação em Educação da Universidade Federal de Minas Gerais. Resolução no 14, de 2 de abril de 2018. Dá nova redação à Resolução 03/2013 que define normas para o funcionamento do Doutorado Latino-Americano em Educação: Políticas Públicas e Profissão Docente. Documento impreso.

UNESCO. Organização das Nações Unidas para a Educação, Ciência e Cultura. Unesco Science Report: towards 2030. Paris: Unesco, 2015.

\section{NOTAS}

1 Los datos se produjeron principalmente en el ámbito de la tesis de doctorado titulada "Itinerários da internacionalização da educaşão superior no âmbito da América Latina e Caribe", de autoría de Juliana de Fátima Souza, presentada en 2018 en el Programa de Pós-Graduação em Educação da UFMG y desarrollada con beca de la Capes. La investigación fue aprobada por el Comité de Ética de la Universidad, según parecer nº 1.114.313 - CAAE 45225615.8.0000.5149. Para este artículo, se han sumado nuevos análisis.

2 Conferencia Mundial sobre Educación para Todos (Jomtien, Tailandia, 1990); el Foro Mundial de Educación (Dakar, Senegal, 2000); las Conferencias de los Ministros de Educación de América Latina y el Caribe; Programa de Educación para América Latina y el Caribe (PRELAC) Habana, Cuba, Noviembre, 2002.

3 Los docentes que participaron de la reunión representando al PPGE/UFMG y que contribuyeron en las articulaciones y concepciones del Doctorado fueron: Dalila Andrade Oliveira, Lucíola Licínio de Castro Paixão Santos, Luiz Alberto Oliveira Gonçalves e Inês Assunção de Castro Teixeira.

4 Información obtenida de forma verbal de la coordinadora de postgrado en educación de la UFMG, profesora María de Fátima Cardoso Gomes, durante la $69^{\circ}$ Reunión anual de la Sociedad Brasilera para el Progreso y la Ciencia, en mesa redonda sobre la "Internacionalización de los cursos de postgrado Sur-Sur", realizada el 21 de julio de 2017 en Belo Horizonte, Video (1h41 min) disponible en <https://www.facebook.com/pensar. aeducacao/videos/1699396667034162/>, acceso 28 de noviembre de 2017.

5 En 2010, de los 98 programas de postgrado en educación del Brasil, 51 ofrecían maestría y doctorado, 46 solamente maestría y solo uno maestría profesional (modalidad ofrecida en Brasil), Las instituciones con calificación CAPES 6 y 7 eran: Universidade Federal de Minas Gerais, Pontifícia Universidade Católica do Rio de Janeiro, Universidade do Estado do Rio de Janeiro, Universidade Federal Fluminense, Pontifícia Universidade Católica do Rio Grande do Sul, Universidade do Vale do Rio dos Sinos, Universidade Federal de São Carlos y Universidade de São Paulo. La UFMG, 
por su parte, obtuvo la nota máxima en las evaluaciones trienales en 2010 y en 2013 y en la evaluación cuadrienal de 2017. Datos disponibles en <https://geocapes.capes.gov.br/ geocapes/>, acceso 23 de noviembre de 2017.

6 Informaciones que constan en la Ficha de Evaluación del Programa de Postgrado en Educación de la UFMG, diligenciada por la CAPES, referente al trienio 2009-2012. Disponible en <http://www.posgrad.fae.ufmg.br/site/wp-content/uploads/2016/12/ Ficha_avaliacao_2013_038_32001010001P7.pdf >, acceso 23 de noviembre de 2017.

7 A lo largo de su existencia, el DLA no conto siempre con apoyo administrativo de forma sistemática. Eso se hace evidente en el testimonio de los estudiantes. Así, muchas demandas y necesidades han sido resueltas por los propios docentes y discentes, frente a las limitaciones que implica una secretaria unificada del PPGE para responder a todas las demandas.

8 Información extraída de la p. 7 de la relatoría Docentes excelentes: Cómo mejorar el aprendizaje en América Latina y el Caribe, de autoria de Barbara Bruns e Javier Luque, publicado por el Banco Mundial en 2014. Disponible en <http://www.bancomundial.org/content/dam/ Worldbank/Highlights $\% 20 \& \% 20$ Features/lac/LC5/Spanish-excellent-teachers-report. pdf $>$. Acceso el 29 de noviembre de 2017.

9 Noticia publicada en la página web de la Capes el 08 de marzo de 2017. Disponible en <http://www.capes.gov.br/sala-de-imprensa/noticias/8315-mulheres-sao-maioria-na-posgraduacao-brasileira>. Acceso el 29 de noviembre de 2017.

10 Como en América Latina y el Caribe, a ejemplo de Chile, hay países en los que la universidad pública no es gratuita, la pregunta hecha a los participantes extranjeros fuer: "¿jel curso fue pago por usted?”.

11 Información verbal de la profesora María de Fátima Cardoso Gomes, coordinadora del PPGE/UFMG, durante la mesa redonda "Historia del Doctorado Latinoamericano en Educación”, realizada en Belo Horizonte el 26 de junio de 2016.

Submetido: 08/02/2019

Aprovado: 25/03/2019

Contacto:

Juliana F. Souza

Rua Miguel Pereira, no 39 - Boa Vista Belo Horizonte | MG | Brasil

CEP 31.060-150

Lívia M. Fraga Vieira

Rua Chile, no 197 - Sion

Belo Horizonte | MG | Brasil

CEP 30.310-670 